\title{
Transverse Rectal Fold
}

National Cancer Institute

\section{Source}

National Cancer Institute. Transverse Rectal Fold. NCI Thesaurus. Code C33807.

One of three or four semi-lunar horizontal folds of the rectal wall that serve to support the bulk of fecal matter. 